\title{
The Stochastic Limit for the Hydrogen Atom in Interaction with the EM Field
}

\author{
L. Accardi · F.G. Cubillo
}

Received: 13 November 2007 / Accepted: 21 January 2008 / Published online: 31 January 2008

(C) Springer Science+Business Media, LLC 2008

\begin{abstract}
In the stochastic limit the resonances play a fundamental role because they determine the generalized susceptivities which are the building blocks of all the physical information which survives in this limit. There are two sources of possible divergences, one related to the singularities of the form factor, another to the chaoticity of the spectrum. The situation will be illustrated starting from the example of the discrete part of the hydrogen atom in interaction with the electromagnetic field.
\end{abstract}

Keywords Stochastic limit of quantum theory $\cdot$ Generalized susceptivities $\cdot$ Hydrogen atom $\cdot$ Electromagnetic field $\cdot$ Form factor

\section{Introduction}

The stochastic limit approach of quantum theory [2,3] starts from the usual quantum Hamiltonian dynamics in interaction representation. Depending such dynamics on a parameter $\lambda$, one rescales some parameters in the associated evolution (typically time) and obtains in the limit $\lambda \rightarrow 0$ a new dynamical system driven by a (singular) white noise Hamiltonian. By the stochastic resonance principle, in the limit we shall have not a single but an infinity of independent quantum noises, one for each suitable Bohr frequency of the system [1].

This allows to estimate the behaviour of a complex system with many degrees of freedom in terms of relatively few functions of the microscopic characteristics of the quickly relaxing degrees of freedom, which introduce quantum mechanical fluctuation-dissipation phenomena in the evolution. According to the interpretation, these functions are called susceptivities, transport coefficients, etc. In the stochastic limit the real part of each generalized

L. Accardi

Centro Vito Volterra, Università degli Studi di Roma "Tor Vergata", 00133 Roma, Italy

e-mail: accardi@ volterra.mat.uniroma2.it

F.G. Cubillo (凶)

Departamento de Análisis Matemático, Universidad de Valladolid, 47005 Valladolid, Spain

e-mail: fgcubill@am.uva.es 
susceptivity is a $\delta$-function and its imaginary part a generalized Hilbert transform, both over each resonant surface, see (9). The evolution corresponding to the real part is a contraction, i.e. dissipative, whereas the imaginary part corresponds to a global shift in the spectrum of the system Hamiltonian.

In this work we illustrate the situation with a concrete physical example: the bounded states of the hydrogen atom in interaction with the electromagnetic field. We obtain some sufficient conditions on the form factors for the existence of the generalized susceptivities and calculate them explicitly.

The paper is organized as follows. Section 2 is an introduction to the stochastic limit of quantum theory. Section 3 includes some technical remarks on distributional calculus and establishes connections between the real and imaginary parts of the generalized susceptivities. Finally, Sect. 4 contains the study of the hydrogen atom in interaction with the electromagnetic field.

\section{The Stochastic Limit Approach}

The general scheme of the stochatic limit technique for a Hamiltonian $H$ with decomposition into free and interacting parts

$$
H^{(\lambda)}=H_{0}+\lambda H_{I},
$$

where $\lambda$ is a coupling constant, considers the Schrödinger equation in interaction representation

$$
\begin{gathered}
\frac{d}{d t} U_{t}^{(\lambda)}=-i \lambda H_{I}(t) U_{t}^{(\lambda)}, \quad U_{0}=I, \\
H_{I}(t):=e^{i t H_{0}} H_{I} e^{-i t H_{0}},
\end{gathered}
$$

whose solution is the propagator $U_{t}^{(\lambda)}=e^{i t H_{0}} e^{-i t H^{(\lambda)}}$.

Then one makes the time rescaling $t \mapsto \lambda^{2} t$ and studies the limits

$$
\lim _{\lambda \rightarrow 0} \frac{1}{\lambda^{2}} H_{I}^{(\lambda)}\left(t / \lambda^{2}\right)=: H_{t}, \quad \lim _{\lambda \rightarrow 0} U_{t / \lambda^{2}}^{(\lambda)}=: U_{t},
$$

in a topology to be specified, as well as the equations satisfied by them.

In what follows we are interested in system-reservoir Hamiltonians of the form

$$
\left\{\mathcal{H}_{S} \otimes \mathcal{H}_{R}, H_{S R}=H_{S} \otimes 1_{R}+1_{S} \otimes H_{R}+\lambda H_{I}\right\}
$$

where $H_{0}=H_{S} \otimes 1_{R}+1_{S} \otimes H_{R}$ is the free Hamiltonian and the interaction Hamiltonian $H_{I}$ contains all the new physics with respect to the isolated systems.

Assume the free system Hamiltonian $H_{S}$ has a non degenerate discrete spectrum,

$$
H_{S}=\sum_{n} \varepsilon_{n} P_{n}=\sum_{n} \varepsilon_{n}\left|\varepsilon_{n}\right\rangle\left\langle\varepsilon_{n}\right|,
$$

and as reservoir $R$ consider a boson quantum field described by a Fock space $\mathcal{F}$ with the vacuum reference vector and a family of operator-valued distributions $a_{k^{\prime}}^{+}, a_{k}$ on $\mathbb{R}^{d}(d \geq 3)$, the creation and annihilation densities, which satisfy the commutation relations $\left[a_{k}, a_{k^{\prime}}^{+}\right]=$ $\delta\left(k-k^{\prime}\right)$. The free Hamiltonian of a bosonic reservoir $R$ has the form

$$
H_{R}=\int \omega_{k} a_{k}^{+} a_{k} d k
$$


where the real-valued function $\omega(k)$ is the free 1-particle Hamiltonian. In this work the functions under consideration shall be $\omega(k)=|k|$ and $\omega(k)=k^{2}$.

For an interaction Hamiltonian $H_{I}$ of dipole-type

$$
H_{I}=\int d k\left\{D_{k} \otimes g_{k} a_{k}^{+}+D_{k}^{+} \otimes \bar{g}_{k} a_{k}\right\}
$$

where $\left\{D_{k}: k \in \mathbb{R}^{d}\right\}$ is the family of response terms or currents (operators acting on $\mathcal{H}_{S}$ ) and the $g_{k}$ are the form factors, the time evolved interaction Hamiltonian becomes

$$
H_{I}(t)=\int d k \sum_{m, n} P_{m} D_{k} P_{n} \otimes g_{k} e^{i t\left(\omega_{k}+\varepsilon_{m}-\varepsilon_{n}\right)} a_{k}^{+}+\text {h.c. }
$$

Introducing the operators

$$
D_{\omega}(k):=\sum_{\varepsilon_{n}-\varepsilon_{m}=\omega} P_{m} D_{k} P_{n}=\sum_{\varepsilon_{n}-\varepsilon_{m}=\omega}\left\langle\varepsilon_{n}\left|D_{k}\right| \varepsilon_{m}\right\rangle\left|\varepsilon_{m}\right\rangle\left\langle\varepsilon_{n}\right|,
$$

the evolved interaction Hamiltonian can be written in the so called standard or canonical form

$$
H_{I}(t)=\int d k \sum_{\omega} D_{\omega}(k) \otimes g_{k} e^{i t\left(\omega_{k}-\omega\right)} a_{k}^{+}+\text {h.c. }
$$

The stochastic limit of the evolution equation in interaction picture for our model leads to the normally ordered stochastic Schrödinger equation

$$
d U_{t}=(-i d H(t)-G d t) U_{t}, \quad t>0,
$$

with initial condition $U_{0}=1$. The first term $d H(t)$ is the martingale term, which won't be considered here. The second term $G d t$, called the drift, is of the form

$$
G=\sum_{\omega} \pi \int_{\mathbb{R}^{d}} d k\left|D_{\omega}(k)\right|^{2}|g(k)|^{2} \delta\left(\omega_{k}-\omega\right)-i \text { P.P. } \int_{\mathbb{R}^{d}} d k \frac{\left|D_{\omega}(k)\right|^{2}|g(k)|^{2}}{\omega_{k}-\omega} .
$$

The integrals are taken over an operator function and therefore they should be interpreted as weak integrals, i.e. as integrals over the matrix elements of the operators $\left|D_{\omega}(k)\right|^{2}$. If for any Bohr frequency $\omega$ there exists a unique pair of energy levels $\varepsilon_{1_{\omega}}, \varepsilon_{2_{\omega}} \in \operatorname{Spec} H_{S}$ such that $\omega=\varepsilon_{2_{\omega}}-\varepsilon_{1_{\omega}}$, then

$$
\left|D_{\omega}(k)\right|^{2}=D_{\omega}(k)^{+} D_{\omega}(k)=\left|\left\langle\varepsilon_{1_{\omega}}|D(k)| \varepsilon_{2_{\omega}}\right\rangle\right|^{2}\left|\varepsilon_{2_{\omega}}\right\rangle\left\langle\varepsilon_{2_{\omega}}\right|
$$

and we can write the drift term as

$$
G=\sum_{\omega} \gamma_{\omega}^{-}\left|\varepsilon_{2_{\omega}}\right\rangle\left\langle\varepsilon_{2_{\omega}}\right|
$$

where the generalized susceptivity factors $\gamma_{\omega}^{-}$are defined by

$$
\begin{aligned}
\gamma_{\omega}^{-} & :=\pi \int_{\mathbb{R}^{d}} d k\left|g_{\omega}(k)\right|^{2} \delta\left(\omega_{k}-\omega\right)-i \mathrm{P} . \mathrm{P} . \int_{\mathbb{R}^{d}} d k \frac{\left|g_{\omega}(k)\right|^{2}}{\omega_{k}-\omega}, \\
g_{\omega}(k) & :=\left\langle\varepsilon_{1_{\omega}}|D(k)| \varepsilon_{2_{\omega}}\right\rangle g(k)
\end{aligned}
$$


and contain all the physical information of the original Hamiltonian system.

The drift term corresponds to a non-selfadjoint correction to the system Hamiltonian that can be considered as the prototype of the quantum mechanical fluctuation-dissipation relation:

$$
i H_{S} \rightarrow i H_{S}-G=i\left(H_{S}-\sum_{\omega} \operatorname{Im}\left(\gamma_{\omega}^{-}\right)\left|\varepsilon_{2_{\omega}}\right\rangle\left\langle\varepsilon_{2_{\omega}}\right|\right)-\sum_{\omega} \operatorname{Re}\left(\gamma_{\omega}^{-}\right)\left|\varepsilon_{2_{\omega}}\right\rangle\left\langle\varepsilon_{2_{\omega}}\right| .
$$

The imaginary part of $G$ is a global shift in the spectrum of the system Hamiltonian. The evolution generated by the real part of $G$ is a contraction, in general nonunitary, i.e. dissipative. Since $\omega(k)>0$, (9) shows that $\operatorname{Re}\left(\gamma_{\omega}^{-}\right)=0$ if $\omega \leq 0$. But (9) also shows that this is not the case for the imaginary part. In the stochastic limit the negative Bohr frequencies contribute with an overall red shift to the energy.

\section{The Distribution $(\omega(k)-\omega)^{-1}$}

Let

$$
k \in \mathbb{R}^{d} \rightarrow \omega(k)-\omega \in \mathbb{R}
$$

be a $C^{\infty}$-function except perhaps for some closed set of singular points with $d$-dimensional Lebesgue measure zero. In this section we study, for each test function $\phi \in \mathcal{D}\left(\mathbb{R}^{d}\right)$, the integral

$$
\left\langle\frac{1}{\omega(k)-\omega}, \phi(k)\right\rangle=\int_{\mathbb{R}^{d}} \frac{\phi(k)}{\omega(k)-\omega} d k
$$

and its Cauchy principal value or principal part

$$
\left\langle\text { P.P. } \frac{1}{\omega(k)-\omega}, \phi(k)\right\rangle=\lim _{\epsilon \rightarrow 0} \int_{|\omega(k)-\omega|>\epsilon} \frac{\phi(k)}{\omega(k)-\omega} d k,
$$

just the imaginary part of the generalized susceptivity factor $\gamma_{-}$, see (9).

To this end, assume that $\nabla \omega(k) \neq 0$ for each regular point $k \in \mathbb{R}^{d}$. Then the equation $\omega(k)-\omega=$ cte defines a regular surface $S_{c}$ of dimension $d-1$ in a neighbourhood sufficiently small of each regular point verifying the equation. In such neighbourhood we can consider a local change of variables $\psi:\left(u_{1}, \ldots, u_{d}\right) \rightarrow\left(k_{1}, \ldots, k_{d}\right)$ such that the surface $S_{c}$ is given by fixing one of the $u$ 's to the value of the constant $c$. Choose, for example, $\omega(k)-\omega=u_{1}$ and arbitrarily the $u_{2}, \ldots, u_{d}$, but with the condition that the Jacobian $J \psi(u)$ is different from zero. Then, by the change of variables theorem, we will have ${ }^{1}$

$$
\int_{\mathbb{R}^{d}} \frac{\phi(k)}{\omega(k)-\omega} d k=\int_{\psi^{-1}\left(\mathbb{R}^{d}\right)} \frac{\phi(\psi(u))}{u_{1}} J \psi(u) d u,
$$

being the last integral, by Fubini's theorem, equal to

$$
\int_{a}^{b} \frac{1}{u_{1}}\left[\int_{\psi_{u_{1}}^{-1}} \phi(\psi(u)) J \psi(u) d u_{2} \cdots d u_{d}\right] d u_{1}
$$

\footnotetext{
${ }^{1}$ We can assume that the support of $\phi$ is contained in the neighbourhood where the local change of variables is given, else we consider a suitable locally finite partition of the unity.
} 
where $\psi^{-1}\left(\mathbb{R}^{d}\right)=\left\{u_{1} \times \psi_{u_{1}}^{-1} \mid u_{1} \in[a, b]\right\}$.

We can write the integral (10) in terms of differential forms. For it, consider the form $\Omega_{c}$ of order $d-1$ associated to the function $W(k)=\omega(k)-\omega$ on the surface $S_{c}$ by the equation

$$
d W \wedge \Omega_{c}=d v,
$$

where $d v=d k_{1} \wedge \cdots \wedge d k_{d}$ is the volume element in $\mathbb{R}^{d}$. The form $\Omega_{c}$ verifying (11) is not unique since we can add to it any form $\Lambda$ orthogonal to $d W$, that is, such that $d W \wedge \Lambda=0$. Such forms $\Lambda$ can be written as $\Lambda=\gamma \wedge d W$, where $\gamma$ is a certain form of order $d-2$. We note also that the form $\Omega_{c}$ does not depend on the choice of coordinates $u_{2}, \ldots, u_{d}$, but it does on the function $W$ defining the surface $S_{c}$. In our case we have

$$
\Omega_{c}=\Omega_{u_{1}}=\left.J \psi(u)\right|_{u_{1}=c} d u_{2} \wedge \cdots \wedge d u_{d}
$$

and from (10) we obtain

$$
\int_{\mathbb{R}^{d}} \frac{\phi(k)}{\omega(k)-\omega} d k=\int_{a}^{b} \frac{1}{u_{1}}\left[\int_{S_{u_{1}}} \phi(\psi(u)) \Omega_{u_{1}}\right] d u_{1} .
$$

In terms of the distribution $\delta\left(W-u_{1}\right)=\delta\left(\omega(k)-\omega-u_{1}\right)$ formula (12) can also be written as ${ }^{2}$

$$
\int_{a}^{b} \frac{1}{u_{1}}\left\langle\delta\left(W-u_{1}\right), \phi\right\rangle d u_{1}
$$

Note that the function

$$
\Phi\left(u_{1}\right)=\int_{S_{u_{1}}} \phi(\psi(u)) \Omega_{u_{1}}
$$

that appears in formula (12) is a test function belonging to $\mathcal{D}(a, b)$. Indeed, the change of variables $\psi$, being bicontinuous, transforms compact sets into compact sets, and vice versa. Moreover, since $\psi$ is a $C^{\infty}$-function, $\phi \circ \psi \in \mathcal{D}\left(\mathbb{R}^{d}\right)$ and then its product with the $C^{\infty}$ function $J \psi$ is also in $\mathcal{D}\left(\mathbb{R}^{d}\right)$. Thus, the integral with respect to $\Omega_{u_{1}}$ of that product extends into a set of finite measure and then is a bounded $C^{\infty}$-function of $u_{1}$ with compact support. Finally, similar arguments can be applied to its derivatives.

The same reasoning can be applied when, instead of $\mathcal{D}\left(\mathbb{R}^{d}\right)$, we consider the Schwartz space $\mathcal{S}\left(\mathbb{R}^{d}\right)$, whenever the Jacobian $J \psi$ and its derivatives are of polynomial growth.

Since $\Phi\left(u_{1}\right) \in \mathcal{D}(a, b)$, the integral

$$
\int_{\mathbb{R}^{d}} \frac{\phi(k)}{\omega(k)-\omega} d k=\int_{a}^{b} \frac{1}{u_{1}} \Phi\left(u_{1}\right) d u_{1}
$$

is improper only at $u_{1}=0$ when $a \leq 0 \leq b$. Then, for the study of the convergence of this integral it is convenient to obtain an asymptotic development of $\Phi\left(u_{1}\right)$ for small values of $u_{1}$. To this end, let us consider the functional depending on the complex parameter $\lambda$

$$
\left\langle W_{+}^{\lambda}, \phi\right\rangle=\int_{W>0} W^{\lambda}(k) \phi(k) d k
$$

\footnotetext{
${ }^{2}$ For a definition of the distribution $\delta\left(W-u_{1}\right)$ see, for example, the Chap. 3 of [4].
} 
If the $C^{\infty}$-function $W$ is such that the equation $W(k)=0$ defines locally a $d-1$ dimensional surface of regular points, then the distribution $W_{+}^{\lambda}$ is meromorphic with singularities the sequence of simple poles [4]

$$
\lambda=-1,-2, \ldots,-n, \ldots
$$

The residue of the function (14) at each of these poles can be expressed by means of the test function $\Phi\left(u_{1}\right)$ defined in formula (13), being the residue at $\lambda=-n$ equal to

$$
\operatorname{Res}\left(\left\langle W_{+}^{\lambda}, \phi\right\rangle, \lambda=-n\right)=\frac{\Phi^{(n-1)}(0)}{(n-1) !}
$$

or, in terms of the distributions $\delta^{(k)}(W)$,

$$
\operatorname{Res}\left(\left\langle W_{+}^{\lambda}, \phi\right\rangle, \lambda=-n\right)=\frac{(-1)^{n-1}}{(n-1) !}\left\langle\delta^{(n-1)}(W), \phi\right\rangle .
$$

The knowledge of these singularities permits us write an asymptotic development of $\Phi\left(u_{1}\right)$ for small values of $u_{1}$. Indeed, in our case [4, Sect. 3.4.5]

$$
\Phi\left(u_{1}\right) \simeq \sum_{n=0}^{\infty} \frac{(-1)^{n}}{n !}\left\langle\delta^{(n)}(W), \phi\right\rangle u_{1}^{n}, \quad \text { for } u_{1} \text { small } .
$$

From these results it is easy to derive the following

Proposition 1 Let $W(k)=\omega(k)-\omega$ be a $C^{\infty}$-function, except perhaps for some closed set $E$ of singular points with d-dimensional Lebesgue measure zero, such that the equation $W(k)=0$ defines a $d-1$ dimensional surface $S_{0}$ of regular points, that is, for each point $k_{0}$ in the surface there exist a neighborhood $V$ of $k_{0}$ in $\mathbb{R}^{d}$ and a local system of coordinates $u_{1}, \ldots, u_{d}$ such that $W(k)=u_{1}$ for every $k \in V$ (for example, when $\left.\nabla W\left(k_{0}\right) \neq 0\right)$, and the same is valid for the equation $W(k)=c$ with $c \in(a, b)$, where $\mathbb{R}^{d} \backslash E=\bigcup_{c \in(a, b)} S_{c}$. Then, given a test function $\phi \in \mathcal{D}\left(\mathbb{R}^{d}\right)$, we have:

(i) When $a \leq 0 \leq b$, the integral

$$
\left\langle\frac{1}{\omega(k)-\omega}, \phi(k)\right\rangle=\int_{\mathbb{R}^{d}} \frac{\phi(k)}{\omega(k)-\omega} d k=\int_{a}^{b} \frac{1}{u_{1}} \Phi\left(u_{1}\right) d u_{1}
$$

is finite if and only if $\langle\delta(\omega(k)-\omega), \phi(k)\rangle=0$.

(ii) When $a \leq 0 \leq b$, the Cauchy principal value

$$
\begin{aligned}
\left\langle\text { P.P. } \frac{1}{\omega(k)-\omega}, \phi(k)\right\rangle & =\lim _{\epsilon \rightarrow 0} \int_{|\omega(k)-\omega|>\epsilon} \frac{\phi(k)}{\omega(k)-\omega} d k \\
& =\lim _{\epsilon \rightarrow 0}\left[\int_{a}^{-\epsilon} \frac{1}{u_{1}} \Phi\left(u_{1}\right) d u_{1}+\int_{\epsilon}^{b} \frac{1}{u_{1}} \Phi\left(u_{1}\right) d u_{1}\right]
\end{aligned}
$$

is always finite.

(Recall that, when $0 \notin[a, b]$, the integrals in (i) and (ii) are always finite.)

Proof Since $\Phi\left(u_{1}\right) \in \mathcal{D}(a, b)$, the asymptotic development (15) of $\Phi\left(u_{1}\right)$ is valid in a neighborhood of $u_{1}=0$ and also we can assume that $a$ and $b$ are finite. 
(i) The unidimensional integral $\int_{a}^{b} \Phi\left(u_{1}\right) / u_{1} d u_{1}$ converges if and only if for any $\alpha>0$ we have $\Phi\left(u_{1}, \omega\right) \simeq u_{1}^{\alpha}$ as $u_{1} \rightarrow 0$. By formula (15), this condition is satisfied if and only if $\langle\delta(\omega(k)-\omega), \phi(k)\rangle=0$.

(ii) It is well known [6, Theorem 1.35] that for a function of the form $\Phi\left(u_{1}\right) / u_{1}, \Phi$ being continuous in a neighborhood of $u_{1}=0$, the integral in the P.P. sense exists.

Corollary 2 Proposition 1 is satisfied also for every $\phi \in \mathcal{S}\left(\mathbb{R}^{d}\right)$ if, in addition, the Jacobian $\mathrm{J} \psi$ and its derivatives are of polynomial growth.

Proof Recall that, if $\phi \in \mathcal{S}\left(\mathbb{R}^{d}\right)$, then $\Phi \in \mathcal{S}(a, b)$ when the Jacobian $J \psi$ and its derivatives are of polynomial growth.

Example 3 For the radiative dispersion $\omega(k)=|k|=\left(\sum_{j=1}^{d} k_{j}^{2}\right)^{1 / 2}$, if we take

$$
u_{1}=\omega(k)-\omega, \quad u_{2}=\theta_{1}, \ldots, u_{d}=\theta_{d-1},
$$

where $\theta_{1}, \ldots, \theta_{d-1}$ are the usual angles in spherical coordinates, we obtain

$$
\Omega_{u_{1}}=d \sigma_{S_{u_{1}+\omega}}
$$

being $d \sigma_{S_{u_{1}+\omega}}$ the Euclidean element of surface for the sphere $S_{u_{1}+\omega}$ with center the origin and radius $u_{1}+\omega$. In this case we have

$$
\int_{\mathbb{R}^{d}} \frac{\phi(k)}{\omega(k)-\omega} d k=\int_{-\omega}^{\infty} \frac{1}{u_{1}}\left[\int_{S_{u_{1}+\omega}} \phi(\psi(u)) d \sigma_{S_{u_{1}+\omega}}\right] d u_{1} .
$$

Let us put $\Phi\left(u_{1}, \omega\right)=\int_{S_{u_{1}+\omega}} \phi(\psi(u)) d \sigma_{S_{u_{1}+\omega}}$.

For $\omega<0$ the integrals of (17) converge because $\Phi\left(u_{1}, \omega\right)$ belongs to $\mathcal{D}(-\omega, \infty)$ as function of $u_{1}$.

If $\omega>0$, we are under the hypothesis of Proposition 1 and then the integrals in (17) converge if and only if $\langle\delta(\omega(k)-\omega), \phi(k)\rangle=0$, and they converge in the P.P. sense for all $\phi \in \mathcal{D}\left(\mathbb{R}^{d}\right)$.

If $\omega=0$, we cannot apply Proposition 1 since the equation $W(k)=\omega(k)=0$ does not define a regular surface else a singular point $k=0$, but in this case the integrals of (17) converge because $\Phi\left(u_{1}, 0\right) \rightarrow 0$ as $u_{1} \rightarrow 0$.

By Corollary 2, the same results are satisfied for $\phi \in \mathcal{S}\left(\mathbb{R}^{d}\right)$.

Example 4 For $\omega(k)=k^{2}=\sum_{j=1}^{d} k_{j}^{2}$, applying again the change of variables (16), now we have

$$
\Omega_{u_{1}}=\frac{1}{2\left(\sum_{j=1}^{d} k_{j}^{2}\right)^{1 / 2}} d \sigma_{S_{\sqrt{u_{1}+\omega}}}=\frac{1}{2 \sqrt{u_{1}+\omega}} d \sigma_{S_{\sqrt{u_{1}+\omega}}} .
$$

Then, in this case we obtain

$$
\int_{\mathbb{R}^{d}} \frac{\phi(k)}{\omega(k)-\omega} d k=\int_{-\omega}^{\infty} \frac{1}{u_{1}}\left[\int_{S_{\sqrt{u_{1}+\omega}}} \frac{\phi(\psi(u))}{2 \sqrt{u_{1}+\omega}} d \sigma_{S_{\sqrt{u_{1}}+\omega}}\right] d u_{1} .
$$


Here, $\Phi\left(u_{1}, \omega\right)=\int_{S_{\sqrt{u_{1}+\omega}}} \frac{\phi(\psi(u))}{2 \sqrt{u_{1}+\omega}} d \sigma_{S_{\sqrt{u_{1}+\omega}}}$ and we can apply the same arguments than in Example 3 to determine the convergence of the integrals in (18). Thus, for $\omega \leq 0$ the integrals of (18) converge for every $\phi \in \mathcal{D}\left(\mathbb{R}^{d}\right)$ and, on the other hand, when $\omega>0$ these integrals converge if and only if $\langle\delta(\omega(k)-\omega), \phi(k)\rangle=0$ and they converge in the P.P. sense for all $\phi \in \mathcal{D}\left(\mathbb{R}^{d}\right)$.

By Corollary 2, the same results are satisfied for $\phi \in \mathcal{S}\left(\mathbb{R}^{d}\right)$.

These results will be of applicability in the physical example considered in the next section.

\section{The Hydrogen Atom in the EM Field}

It is well known [5] that in a central potential, caused here by the hydrogen nucleus, the bound states of a spinless electron are determined by three quantum numbers $n, l$ and $m$. The total or energy quantum number $n$, whose range of values is $n=1,2,3, \ldots,+\infty$, determines the energy $E_{n}$ of the electron

$$
E_{n}=-\frac{1}{2} \frac{Z m e^{4}}{n^{2} \hbar^{2}}=-\frac{Z e^{2}}{2 a_{0} n^{2}}
$$

where $m$ and $e$ are the mass and charge of the electron, $a_{0}=\hbar^{2} /\left(m e^{2}\right)$ is the Bohr radius and $Z=1$ for the hydrogen atom. The orbital and magnetic quantum numbers, $l$ and $m$, determine the angular momentum and the angular momentum along the axis of quantization, respectively, and their ranges of values are $l=0,1,2, \ldots, n-1$ and $m=-l,-l+1, \ldots,+l$.

In the following $q$ shall denote the position of the electron in the 3-dimensional space and we shall assume that the nucleus of the hydrogen atom is fixed at the origin, so that, in spherical coordinates, the associated total eigenfunctions $\psi_{n l m}$ are given by

$$
\psi_{n l m}(|q|, \theta, \phi)=R_{n l}(|q|) Y_{l m}(\theta, \phi),
$$

being $Y_{l m}$ the spherical harmonics of order $l$ and $R_{n l}$ the radial eigenfunction corresponding to the quantum numbers $n$ and $l$,

$$
R_{n l}(|q|)=-\left[\left(\frac{2}{n a_{0}}\right)^{3} \frac{(n-l-1) !}{2 n[(n+l) !]^{3}}\right]^{1 / 2} e^{-|q| / 2}|q|^{l} L_{n+l}^{2 l+1}(|q|),
$$

where $L_{n+l}^{2 l+1}$ is the associated Laguerre polynomial

$$
L_{n+l}^{2 l+1}(|q|)=\sum_{s=0}^{n-l-1} \frac{(-1)^{s+2 l+1}[(n+l) !]^{2}|q|^{s}}{(n-l-1-s) !(2 l+1+s) ! s !} .
$$

We shall consider only states of the electron with orbital number $l=0$. Recall that $Y_{00}(\theta, \phi)=(4 \pi)^{-1 / 2}$.

For the interaction of the spinless electron with a nonrelativistic quantum electromagnetic (EM) field, neglecting the quadratic term in the potential $A$ of the field, one can write the interaction Hamiltonian in the form [2]

$$
H_{I}=-\frac{e}{m c} \sum_{\sigma=1,2} \int d^{3} k\left[a_{\sigma}^{+}(k) g_{\sigma}(k) e^{-i k \cdot q}+a_{\sigma}(k) \bar{g}_{\sigma}(k) e^{i k \cdot q}\right] p_{\sigma},
$$


where $k \in \mathbb{R}^{3}$ corresponds to momentum coordinates, $\sigma=1,2$ is a polarization index and $a_{\sigma}$ and $p_{\sigma}$ are Boson annihilators and the momentum of the electron in the corresponding polarization direction, respectively. The usual form factors are $g_{\sigma}(k)=|k|^{-1 / 2}$. The polaron Hamiltonian is obtained by omitting the product with the momentum operator $p$. Thus, for the polaron Hamiltonian the response terms are of the form

$$
D_{\sigma}(k)=e^{i k \cdot q} .
$$

In what follows the polarization index will be neglected and we will use the following notation. For the positive Bohr frequencies we shall write, see (19),

$$
\omega_{m n}:=E_{m}-E_{n}=-\frac{e^{2}}{2 a_{0}}\left(\frac{1}{m^{2}}-\frac{1}{n^{2}}\right), \quad m>n,
$$

and we shall put

$$
\begin{aligned}
g_{m n}(k) & :=g(k)\left\langle\psi_{m 00}, D(k) \psi_{n 00}\right\rangle \\
& =g(k)\left\langle R_{m 0}(|q|) Y_{00}(\theta, \phi), g(k) D(k) R_{m 0}(|q|) Y_{00}(\theta, \phi)\right\rangle .
\end{aligned}
$$

Now suppose that for the positive Bohr frequency $\omega_{m n}$ there exists a unique pair of energy levels $\varepsilon_{m}=E_{m}, \varepsilon_{n}=E_{n}$ in Spec $H_{S}$ such that $\omega_{m n}=E_{m}-E_{n}$. (This is not the case for every positive Bohr frequency $\omega_{m n}$ of the hydrogen atom, but by means of a little perturbation the hydrogen atom becomes a system verifying this generic assumption.) Then, the product of the form factor $g(k)$ by the operator $D_{\omega_{m n}}$ defined by (6) can be written as

$$
g(k) D_{\omega_{m n}}(k)=g_{m n}(k)\left|\psi_{n 00}\right\rangle\left\langle\psi_{m 00}\right|
$$

and the corresponding generalized susceptivity factor $\gamma_{\omega_{m n}}^{-}$is of the form, see (9),

$$
\gamma_{\omega_{m n}}^{-}=\pi \int d k\left|g_{m n}(k)\right|^{2} \delta\left(\omega(k)-\omega_{m n}\right)-i \text { P.P. } \int d k \frac{\left|g_{m n}(k)\right|^{2}}{\omega(k)-\omega_{m n}} .
$$

In what follows we shall study the generalized Hilbert transform that defines the imaginary part of $\gamma_{\omega_{m n}}^{-}$in terms of the form factor $g$. (The real part doesn't present any problem.) We shall restrict our attention to the dispersion functions of Examples 3 and 4,

$$
\omega(k)=|k| \text { and } \quad \omega(k)=k^{2} .
$$

The form factors under consideration will be of the form

$$
g(k):=g(|k|),
$$

being the function $g$, at first, a measurable function of $|k|$. Recall that the usual choice is $g(k)=|k|^{-1 / 2}$.

At first we calculate the matrix elements $g_{m n}$.

Lemma 5 For the interaction of a spinless electron in the hydrogen atom with an electromagnetic field, with form factor of the form (27), we have

$$
g_{m n}(k)=\frac{i g(|k|)}{2|k|} \sum_{s=2}^{m+n} C_{s}^{m n}\left(\frac{1}{(1+i|k|)^{s}}-\frac{1}{(1-i|k|)^{s}}\right),
$$


where

$$
C_{s}^{m n}:=\frac{(-1)^{s} 4}{s a_{0}^{3}(m n)^{3 / 2}} \sum_{\alpha=\max \{0, s-m-1\}}^{\min \{n-1, s-2\}}\left(\begin{array}{c}
n-1 \\
\alpha
\end{array}\right)\left(\begin{array}{c}
m-1 \\
s-2-\alpha
\end{array}\right)\left(\begin{array}{c}
s \\
\alpha+1
\end{array}\right) .
$$

Proof Recall that $Y_{00}(\theta, \phi)=(4 \pi)^{-1 / 2}$ and assume that $k$ is oriented along the positive $q_{3}$-axis. Then,

$$
\begin{aligned}
g_{m n}(k) & =\frac{1}{4 \pi} \int_{0}^{\infty} d|q| \overline{R_{m 0}(|q|)} R_{n 0}(|q|)|q|^{2} \int_{0}^{2 \pi} d \phi \int_{0}^{\pi} d \theta g(|k|) e^{i|k| \cdot|q| \cdot \cos (\theta)} \sin (\theta) \\
& =\frac{i g(|k|)}{2|k|} \int_{0}^{\infty} d|q| R_{m 0}(|q|) R_{n 0}(|q|)|q|\left(e^{-i|k| \cdot|q|}-e^{i|k| \cdot|q|}\right)
\end{aligned}
$$

Denoting by $N_{n 0}$ the normalizing factor for $R_{n 0}$ given in (21), i.e.

$$
N_{n 0}:=-\left[\left(\frac{2}{n a_{0}}\right)^{3} \frac{(n-1) !}{2 n[(n) !]^{3}}\right]^{1 / 2},
$$

the equality (30) becomes

$$
\begin{aligned}
g_{m n}(k) & =N_{m 0} N_{n 0} \frac{i g(|k|)}{2|k|} \int_{0}^{\infty} L_{m}^{1}(|q|) L_{n}^{1}(|q|)|q|\left(e^{-|q|(1+i|k|)}-e^{-|q|(1-i|k|)}\right) d|q| \\
& =\frac{i g(|k|)}{2|k|}[\mathcal{L}(1+i|k|)-\mathcal{L}(1-i|k|)],
\end{aligned}
$$

where $\mathcal{L}(1 \pm i|k|)$ denotes the Laplace transform (see, for example, [6]) at the point $1 \pm i|k|$ of the function $N_{m 0} N_{n 0} L_{m}^{1}(|q|) L_{n}^{1}(|q|)|q|$.

Being $N_{m 0} N_{n 0} L_{m}^{1}(|q|) L_{n}^{1}(|q|)|q|$ a polynomial, the abscissa of summability for its Laplace transform is $a=0$, that is, $\mathcal{L}(z)$ is well defined and holomorphic over the complex semi plane $\Re(z)>0$, in particular, at the points of the form $z=1 \pm i|k|$.

It is well known that $\mathcal{L}_{x^{n} / n !}(z)=1 / z^{n+1}$. From (22) and (31), we have

$$
N_{n 0} L_{n}^{1}(|q|)=\sum_{s=0}^{n-1} \frac{(-1)^{s} 2(n-1) !}{\left(n a_{0}\right)^{3 / 2}(n-1-s) !(s+1) ! s !}|q|^{s} .
$$

After some calculations we obtain

$$
\mathcal{L}(1 \pm i|k|)=\sum_{s=2}^{m+n} \frac{C_{s}^{m n}}{(1 \pm i|k|)^{s}}
$$

where the coefficients $C_{s}^{m n}$ are given by

$$
\begin{aligned}
C_{s}^{m n}= & (s-1) !(-1)^{s-2} \frac{2(m-1) !}{\left(m a_{0}\right)^{3 / 2}} \frac{2(n-1) !}{\left(n a_{0}\right)^{3 / 2}} \\
& \times \sum_{\alpha, \beta} \frac{1}{(m-1-\beta) !(\beta+1) ! \beta !} \frac{1}{(n-1-\alpha) !(\alpha+1) ! \alpha !},
\end{aligned}
$$


the sum taken over the set $\{(\alpha, \beta) \mid \alpha+\beta=s-2,0 \leq \alpha \leq n-1,0 \leq \beta \leq m-1\}$. Substituting (33) in (32) we will have the expression (28).

The change of variables (16) gives us a more convenient expression of the right hand side of (26).

Lemma 6 Let $\omega_{m n}$ be the positive Bohr frequencies given in (24) and $g_{m n}(k)$ the matrix elements given in (28). Then, for the dispersion function $\omega(k)=|k|$, we have

$$
\begin{aligned}
\text { P.P. } & \int_{\mathbb{R}^{3}} \frac{\left|g_{m n}(k)\right|^{2}}{\omega(k)-\omega_{m n}} d k \\
= & \text { P.P. } \int_{-\omega_{m n}}^{\infty} \frac{\pi\left|g\left(u_{1}+\omega_{m n}\right)\right|^{2}}{u_{1}} \\
& \times\left|\sum_{s=2}^{m+n} C_{s}^{m n}\left(\frac{1}{\left(1+i\left(u_{1}+\omega_{m n}\right)\right)^{s}}-\frac{1}{\left(1-i\left(u_{1}+\omega_{m n}\right)\right)^{s}}\right)\right|^{2} d u_{1} .
\end{aligned}
$$

And, for the dispersion function $\omega(k)=k^{2}$, we have

$$
\begin{aligned}
\text { P.P. } & \int_{\mathbb{R}^{3}} \frac{\left|g_{m n}(k)\right|^{2}}{\omega(k)-\omega_{m n}} d k \\
= & \text { P.P. } \int_{-\omega_{m n}}^{\infty} \frac{\pi\left|g\left(\sqrt{u_{1}+\omega_{m n}}\right)\right|^{2}}{2 u_{1} \sqrt{u_{1}+\omega_{m n}}} \\
& \times\left|\sum_{s=2}^{m+n} C_{s}^{m n}\left(\frac{1}{\left(1-i \sqrt{u_{1}+\omega_{m n}}\right)^{s}}-\frac{1}{\left(1-i \sqrt{u_{1}+\omega_{m n}}\right)^{s}}\right)\right|^{2} d u_{1} .
\end{aligned}
$$

Proof For the dispersion function $\omega(k)=|k|$, with the change of variables given in (16), by (17) and since $d \sigma_{S_{r}}=r^{2} d \sigma_{S_{1}}$ in $\mathbb{R}^{3}$, the left hand side of (34) becomes

$$
\text { P.P. } \int_{-\omega_{m n}}^{\infty} \frac{\left(u_{1}+\omega_{m n}\right)^{2}}{u_{1}}\left[\int_{S_{u_{1}+\omega_{m n}}}\left|g_{m n}(k)\right|^{2} d \sigma_{S_{1}}\right] d u_{1} \text {. }
$$

By (28), being the area of $S_{1}$ in $\mathbb{R}^{3}$ equal to $4 \pi$, the integrals over the spheres are

$$
\begin{aligned}
\int_{S_{u_{1}+\omega_{m n}}}\left|g_{m n}(k)\right|^{2} d \sigma_{S_{1}} \\
=4 \pi \mid \frac{i g\left(u_{1}+\omega_{m n}\right)}{2\left(u_{1}+\omega_{m n}\right)} \\
\quad \times\left.\sum_{s=2}^{m+n} C_{s}^{m n}\left(\frac{1}{\left(1-i\left(u_{1}+\omega_{m n}\right)\right)^{s}}-\frac{1}{\left(1-i\left(u_{1}+\omega_{m n}\right)\right)^{s}}\right)\right|^{2} .
\end{aligned}
$$

Substituting this expression in (36) we obtain (34). 
In a similar way, for the dispersion function $\omega(k)=k^{2}$, now by (18), the left hand side of (35) becomes

$$
\text { P.P. } \int_{-\omega_{m n}}^{\infty} \frac{\left(\sqrt{u_{1}+\omega_{m n}}\right)^{2}}{2 u_{1} \sqrt{u_{1}+\omega_{m n}}}\left[\int_{S_{\sqrt{u_{1}+\omega_{m n}}}}\left|g_{m n}(k)\right|^{2} d \sigma_{S_{1}}\right] d u_{1} .
$$

By (28), being the area of $S_{1}$ in $\mathbb{R}^{3}$ equal to $4 \pi$, the integrals over the spheres are

$$
\begin{aligned}
\int_{S_{\sqrt{u_{1}+\omega_{m n}}}}\left|g_{m n}(k)\right|^{2} d \sigma_{S_{1}} \\
=4 \pi \mid \frac{i g\left(\sqrt{u_{1}+\omega_{m n}}\right)}{2 \sqrt{u_{1}+\omega_{m n}}} \\
\times\left.\sum_{s=2}^{m+n} C_{s}^{m n}\left(\frac{1}{\left(1+i \sqrt{u_{1}+\omega_{m n}}\right)^{s}}-\frac{1}{\left(1-i \sqrt{u_{1}+\omega_{m n}}\right)^{s}}\right)\right|^{2} .
\end{aligned}
$$

Substituting this expression in (38) we obtain (35).

We can already determine sufficient conditions on the form factor $g$ in order that the imaginary part of the generalized susceptivity factor $\gamma_{\omega_{m n}}^{-}$exist.

Theorem 7 Let us consider the interaction of a spinless electron in the hydrogen atom with an electromagnetic field, with form factor of the form (27). Then, for the dispersion function $\omega(k)=|k|$, the Cauchy Principal Values

$$
\text { P.P. } \int_{\mathbb{R}^{3}} \frac{\left|g_{m n}(k)\right|^{2}}{\omega(k)-\omega_{m n}} d k, \quad m>n \in \mathbb{N},
$$

are finite if the function $g$ verify the following conditions:

(a1) $\left|g\left(u_{1}+\omega_{m n}\right)\right|^{2} / u_{1}^{7} \in L^{1}([b, \infty))$, with respect to $u_{1}$, for some $b>0$; for example, if $\left|g\left(u_{1}+\omega_{m n}\right)\right| \simeq u_{1}^{v}$ as $u_{1} \rightarrow \infty$, for $v<3$

(a2) the integrand of the right hand side of (34), in a neighborhood of $u_{1}=0$, is the sum of an antisymmetric function $f_{1}$ and a symmetric function $f_{2}$ such that $\int_{\rightarrow 0}^{\epsilon} f_{2}\left(u_{1}\right) d u_{1}$ is finite for some $\epsilon>0$; for example, when $g$ is bounded in a neighborhood of $\omega_{m n}$ (or, equivalently, $g\left(u_{1}+\omega_{m n}\right)$ is bounded for $u_{1}$ in a neighborhood of 0$)$;

(a3) $\left|g\left(u_{1}+\omega_{m n}\right)\right|^{2}\left(u_{1}+\omega_{m n}\right)^{2} \in L^{1}\left(\left[-\omega_{m n},-\omega_{m n}+\epsilon\right]\right)$ with respect to $u_{1}$, for some $\epsilon<$ $\omega_{m n}$; for example, if $\left|g\left(u_{1}+\omega_{m n}\right)\right| \simeq\left(u_{1}+\omega_{m n}\right)^{v}$ as $u_{1} \rightarrow-\omega_{m n}$, for $v>-3 / 2$;

(a4) $g\left(u_{1}+\omega_{m n}\right) \in L^{2}\left(\left[-\omega_{m n}+\epsilon,-\epsilon\right] \cup[\epsilon, b]\right)$, with respect to $u_{1}$, for some $0<\epsilon<\omega_{m n}$ and any finite $b>\epsilon$.

And, for the dispersion function $\omega(k)=k^{2}$, the Principal Parts (40) are finite if the function $g$ verify the following conditions:

(b1) $\left|g\left(\sqrt{u_{1}+\omega_{m n}}\right)\right|^{2} / u_{1}^{9 / 2} \in L^{1}([b, \infty))$, with respect to $u_{1}$, for some $b>0$; for example, if $\left|g\left(\sqrt{u_{1}+\omega_{m n}}\right)\right| \simeq u_{1}^{v}$ as $u_{1} \rightarrow \infty$, for $v<7 / 4$;

(b2) the integrand of the right hand side of (35), in a neighborhood of $u_{1}=0$, is the sum of an antisymmetric function $f_{1}$ and a symmetric function $f_{2}$ such that $\int_{\rightarrow 0}^{\epsilon} f_{2}\left(u_{1}\right) d u_{1}$ is finite for some $\epsilon>0$; for example, when $g$ is bounded in a neighborhood of $\omega_{m n}$ (or, equivalently, $g\left(u_{1}+\omega_{m n}\right)$ is bounded for $u_{1}$ in a neighborhood of 0$)$; 
(b3) $\left|g\left(\sqrt{u_{1}+\omega_{m n}}\right)\right|^{2}\left(u_{1}+\omega_{m n}\right)^{1 / 2} \in L^{1}\left(\left[-\omega_{m n},-\omega_{m n}+\epsilon\right]\right)$ with respect to $u_{1}$, for some $\epsilon<\omega_{m n}$; for example, if $\left|g\left(\sqrt{u_{1}+\omega_{m n}}\right)\right| \simeq\left(u_{1}+\omega_{m n}\right)^{v}$ as $u_{1} \rightarrow-\omega_{m n}$, for $v>-3 / 4$;

(b4) $g\left(\sqrt{u_{1}+\omega_{m n}}\right) \in L^{2}\left(\left[-\omega_{m n}+\epsilon,-\epsilon\right] \cup[\epsilon, b]\right)$, with respect to $u_{1}$, for some $0<\epsilon<\omega_{m n}$ and any finite $b>\epsilon$.

Proof For $y \in \mathbb{R}$ and $C_{s}^{m n} \in \mathbb{R}$, we have

$$
\begin{aligned}
& \sum_{s=2}^{m+n} C_{s}^{m n}\left(\frac{1}{(1+i y)^{s}}-\frac{1}{(1-i y)^{s}}\right) \\
& =\sum_{s=2}^{m+n} \frac{C_{s}^{m n}}{\left(1+y^{2}\right)^{s}}\left[(1-i y)^{s}-(1+i y)^{s}\right] \\
& =\sum_{s=2}^{m+n} \frac{C_{s}^{m n}}{\left(1+y^{2}\right)^{s}} 2 i \sum_{j=0}^{\left[\frac{s-1}{2}\right]}\left(\begin{array}{c}
s \\
2 j+1
\end{array}\right)(-1)^{j+1} y^{2 j+1},
\end{aligned}
$$

where $\left[\frac{s-1}{2}\right]$ denotes the integer part of $\frac{s-1}{2}$. Moreover, the modulus square of (41) is equal to

$$
\sum_{s, s^{\prime}=2}^{m+n} \frac{4 C_{s}^{m n} C_{s^{\prime}}^{m n}}{\left(1+y^{2}\right)^{s+s^{\prime}}} \sum_{j=0}^{\left[\frac{s-1}{2}\right]} \sum_{j^{\prime}=0}^{\left[\frac{s^{\prime}-1}{2}\right]}\left(\begin{array}{c}
s \\
2 j+1
\end{array}\right)\left(\begin{array}{c}
s^{\prime} \\
2 j^{\prime}+1
\end{array}\right)(-1)^{j+j^{\prime}} y^{2\left(j+j^{\prime}+1\right)} .
$$

For the dispersion function $\omega(k)=|k|$, by (42) with $y=u_{1}+\omega_{m n}$, the summands in the integrand of the right hand side of (34), save a constant factor, are of the form

$$
\frac{\left|g\left(u_{1}+\omega_{m n}\right)\right|^{2}\left(u_{1}+\omega_{m n}\right)^{2 l+2}}{u_{1}\left(1+\left(u_{1}+\omega_{m n}\right)^{2}\right)^{r}}, \quad 4 \leq r \leq 2(m+n), 0 \leq l \leq \frac{r-\rho}{2},
$$

where $\rho=2$ if $s, s^{\prime}$ are both odd, $\rho=3$ if one of the $s, s^{\prime}$ is odd and the other even, $\rho=4$ if $s, s^{\prime}$ are both even.

Thus, at $u_{1}=\infty$ the summands with worse behavior are equivalent, save a constant factor, to $\left|g\left(u_{1}+\omega_{m n}\right)\right|^{2} / u_{1}^{7}$ (those for $r=4,5,6$ and $l=\frac{r-\rho}{2}$ ); from this we obtain the condition (a1). At $u_{1}=0$ the divergence due to the factor $1 / u_{1}$ must be canceled by taking the Cauchy Principal Value; then, we must have the condition (a2). At $u_{1}=-\omega_{m n}$ the summands with worse behavior (those with $l=0$ ) are equivalent, save a constant factor, to $\left|g\left(u_{1}+\omega_{m n}\right)\right|^{2}\left(u_{1}+\omega_{m n}\right)^{2}$; then, for the convergence of the integral at $u_{1}=-\omega_{m n}$ the condition (a3) must be verified. Finally, at any other point of the domain of integration we have $\alpha<\left|\frac{\left(u_{1}+\omega_{m n}\right)^{2 l+2}}{u_{1}\left(1+\left(u_{1}+\omega_{m n}\right)^{2}\right)^{r}}\right|<\beta$ for some $\alpha, \beta>0$; then, for the convergence of the integral the condition (a4) is sufficient.

On the other hand, for the dispersion function $\omega(k)=k^{2}$, by (42) now with $y=$ $\sqrt{u_{1}+\omega_{m n}}$, the summands in the integrand of the right hand side of (35), save a constant factor, are of the form

$$
\frac{\left|g\left(\sqrt{u_{1}+\omega_{m n}}\right)\right|^{2}\left(u_{1}+\omega_{m n}\right)^{l+1 / 2}}{u_{1}\left(1+u_{1}+\omega_{m n}\right)^{r}}, \quad 4 \leq r \leq 2(m+n), 0 \leq l \leq \frac{r-\rho}{2} .
$$

In this case, at $u_{1}=\infty$ the summands with worse behavior (those for $l=\frac{r-\rho}{2}$ ) are equivalent, save a constant factor, to $\left|g\left(\sqrt{u_{1}+\omega_{m n}}\right)\right|^{2} / u_{1}^{9 / 2}$; from this we have the condition 
(b1). At $u_{1}=0$ the divergence due to the factor $1 / u_{1}$ must be canceled by taking the Cauchy Principal Value; then, we must have the condition (b2). At $u_{1}=-\omega_{m n}$ the summands with worse behavior (those with $l=0$ ) are equivalent, save a constant factor, to $\left|g\left(\sqrt{u_{1}+\omega_{m n}}\right)\right|^{2}\left(u_{1}+\omega_{m n}\right)^{1 / 2}$; then, for the convergence of the integral at $u_{1}=-\omega_{m n}$ the condition (b3) must be verified. Finally, at any other point of the domain of integration we have $\alpha<\left|\frac{\left(u_{1}+\omega_{m n}\right)^{l+1 / 2}}{u_{1}\left(1+u_{1}+\omega_{m n}\right)^{r}}\right|<\beta$ for some $\alpha, \beta>0$; then, for the convergence of the integral the condition (b4) is sufficient.

Note that conditions (a2) and (b2) imply some special behavior of the form factor $g$ in a neighborhood of the resonance surface $u_{1}=\omega(k)-\omega_{m n}=0$.

Corollary 8 Under the conditions of Proposition 7, we have:

(i) For the dispersion function $\omega(k)=|k|$, the Principal Parts (40) are finite if the form factor $g(|k|)$ verify the following conditions:

(i1) When $|k| \rightarrow \infty,|g(|k|)| \simeq|k|^{v}$, with $v<3$.

(i2) At $|k|=0,|g(|k|)| \simeq|k|^{v}$, with $v>-3 / 2$.

(i3) $g(|k|)$ is bounded elsewhere.

(ii) For the dispersion function $\omega(k)=k^{2}$, the Principal Parts (40) are finite if the form factor $g(|k|)$ verify the following conditions:

(ii1) When $|k| \rightarrow \infty,|g(|k|)| \simeq|k|^{v}$, with $v<7 / 2$.

(ii2) $A t|k|=0,|g(|k|)| \simeq|k|^{v}$, with $v>-3 / 2$.

(ii3) $g(|k|)$ is bounded elsewhere.

Proof The conditions (a1), (a3), (b1) and (b3) of Theorem 7 lead to the conditions (i1), (i2), (ii1) and (ii2), respectively. The conditions (i3) and (ii3) can be derived from the other conditions of Theorem 7 .

Corollary 9 Let us consider the interaction of a spinless electron in the hydrogen atom with an electromagnetic field, with form factor of the form

$$
g(k)=\frac{1}{|k|^{\mu}}, \quad \mu>0 .
$$

Then, for the dispersion functions $\omega(k)=|k|$ and $\omega(k)=k^{2}$, the Principal Parts (40) are finite if and only if $\mu<3 / 2$.

Proof In this case, the conditions (i1), (i3), (ii1) and (ii3) of Corollary 8 are satisfied for all $\mu>0$, and the conditions (i2) and (ii2) are necessary and sufficient.

Recall that the usual choice is $g(k)=|k|^{-1 / 2}$, that is $\mu=1 / 2$.

Acknowledgements F.G. Cubillo wishes to acknowledge financial support from Centro Vito Volterra (Italy), JCyL-project VA013C05 (Castilla y León) and MEC-project FIS2005-03989 (Spain).

\section{References}

1. Accardi, L., Cubillo, F.G.: Master fields, drift and dispersion in the stochastic limit of quantum theory. Open Syst. Inf. Dyn. 14, 459-477 (2007) 
2. Accardi, L., Lu, Y.G., Volovich, I.: Quantum Theory and Its Stochastic Limit. Springer, Berlin (2002)

3. Accardi, L., Kozyrev, S.V.: Quantum interacting particle systems (Lectures given by the authors at the Volterra-CIRM International School). In: Quantum Interacting Particle Systems, Levico Terme, 23-29 September 2000, pp. 1-193. World Scientific, Singapore (2002)

4. Gelfand, I.M., Shilov, G.E.: Les Distributions. Dunod, Paris (1962)

5. Pauling, L., Wilson, E.B. Jr.: Introduction to Quantum Mechanics. McGraw-Hill, New York (1935)

6. Schwartz, L.: Méthodes Mathématiques pour les Sciences Physiques. Hermann, Paris (1966) 